\title{
TIME DELAY SYSTEMS WITH DISTRIBUTION DEPENDENT DYNAMICS
}

\author{
H. T. Banks ${ }^{a}{ }^{\star}, \dagger$, Sava Dediu ${ }^{a, b, \ddagger}$, and Hoan K. Nguyena,§ \\ a Center for Research in Scientific Computation, Box 8205, North Carolina State University, Raleigh, \\ N.C. 27695-8205 USA \\ b Statistical and Applied Mathematical Sciences Institute, 19 T. W. Alexander Dr, P. O. Box 14006, \\ Research Triangle Park, NC 27709-4006, USA
}

\begin{abstract}
General delay dynamical systems in which uncertainty is present in the form of probability measure dependent dynamics are considered. Several motivating examples arising in biology are discussed. A functional analytic framework for investigating well-posedness (existence, uniqueness and continuous dependence of solutions), inverse problems, sensitivity analysis and approximations of the measures for computational purposes is surveyed.
\end{abstract}

\section{Keywords \\ Inverse dynamics problem; Probability distribution function; Sensitivity analysis; Biomedical systems}

\section{INTRODUCTION}

The purpose of this presentation is to survey recent as well as forthcoming results in our research efforts on models with delays and hysteresis where probabilistic uncertainty is present in a significant way. While we focus our motivation here on examples arising in biological applications (Banks and Bihari, 2001; Banks and Holte, 2003; Banks and Potter, 2003; Banks and Bortz, 2005a; Banks and Bortz, 2005b; Banks and Davis, to appear; Banks and Pinter, 2005; Banks and Allnutt, to appear; Banks and Nguyen, to appear; Banks and Nguyen, 2006), similar systems arise in other applications as diverse as materials (Banks and Medhin, submitted; Banks and Webb, 1997a; Banks and Webb, 1997b; Banks and Pinter, 2004; Banks and Pinter, to appear; Banks and Pinter, submitted; Banks and Pinter, 2005), electromagnetics (Banks and Gibson, 2005; Banks and Gibson, to appear), physics, communication networks, etc. As is explained here, there are a wide class of models related to cellular level population dynamics that lead to systems of the form

\footnotetext{
*htbanks@ncsu.edu,

$\dagger^{\dagger}$ Plenary Lecture, $6^{\text {th }}$ IFAC Workshop on Time Delay Systems L'Aquila, IT, July 10-13, 2006

\$sdediu@ncsu.edu

\$hknguyen@ncsu.edu
}

Publisher's Disclaimer: This is a PDF file of an unedited manuscript that has been accepted for publication. As a service to our customers we are providing this early version of the manuscript. The manuscript will undergo copyediting, typesetting, and review of the resulting proof before it is published in its final citable form. Please note that during the production process errors may be discovered which could affect the content, and all legal disclaimers that apply to the journal pertain. 


$$
\dot{x}(t)=\int_{-r}^{0} x(t+\theta) d P(\theta)+f(t, x(t))
$$

where $P$ is a generally unknown probability measure that must be estimated from aggregate or population level (as opposed to individual level) observations or data. The probability measure $P$ (which we shall also refer to as a probability distribution when no confusion results) may be discrete, absolutely continuous (continuous) or a combination of both. In addition to the obvious inverse problems, there are fundamental questions related to modeling of uncertainty, well-posedness, sensitivity, estimation and approximation. The primary goal of this note is to outline a theoretical and computational framework to treat these problems.

\section{EXAMPLE FROM CELLULAR PATHWAYS: HIV INFECTION}

Our first example is typical of delay systems that arise in biochemical pathways and cellular level kinetics of drug metabolism as well as other synthesis models. In (Banks and Holte, 2003; Banks and Bortz, 2005a) the authors study a model for progression of Human Immunodeficiency Virus (HIV) at the cellular level. The model involves compartments $T, A$, $C$, and $V$ for in vitro blood level counts in mice of target (CD4+) cells, acutely infected cells, chronically infected cells and active viral particles, respectively. Free virus $V$ infects target cells $T$, transforming them into acutely infected cells $A$ which at some time later become chronically infected cells $C$. The basic pathway for infection and production of virus for acutely infected cells is schematically depicted in Figure 1. For models in which the individual kinetics for loss of envelope and capsid, integration, transcription, and assembly are not detailed, it is necessary (see (Banks and Holte, 2003)) to include a delay $\tau_{1}$ from the time of infection of a target cell $T$ until it first produces free virus $V$. There is also some delay $\tau_{2}$ before an acutely infected cell $A$ becomes a chronically infected cell $C$.

Here we outline a brief derivation from first principles (with assumptions based on the biology) that supports a mathematical model in which the delays are treated as probabilistically distributed across the population of cells found in a typical in vitro culture.

First consider the delay between initial acute infection and the cell becoming what is termed a chronically infected cell characterized by differences in cell dynamics (see (Banks and Holte, 2003)). It is biologically unrealistic (and unacceptable in the modeling to biologists) to expect an entire population of cells to simultaneously change infection characteristics precisely $\tau_{2}$ $\left(\tau_{2}>0\right)$ hours after initial viral infection. Therefore, one might suppose that the delay between initial acute infection and chronic infection varies across the cell population (thus mathematically characterizing the intercellular variability) according to a probability distribution $\overline{P_{2}}$ (which is not assumed to necessarily possess a density $\overline{p_{2}}$ - it could have point masses). Denote by $C(t ; \tau)$ the subpopulation consisting of chronically infected cells that either maintained their acute infection characteristics for $\tau$ time units or are the progeny of those same cells. In other words, for some $\tau>0$, there exists a subpopulation $C(t ; \tau)$ of the chronically infected cells which either spent $\tau$ hours as acutely infected cells (before converting to chronically infected cells) or are descendants of cells that spent exactly $\tau$ hours as acutely infected cells. Thus, the rate of change in this subpopulation of cells is governed by

$\dot{C}(t ; \tau)=\left(r_{v}-\delta_{c}-\delta X(t)\right) C(t ; \tau)+\gamma A(t-\tau)$,

where 
$X(t)=A(t)+C(t)+T(t)$

is the total number of $C D 4+$ cells (infected and uninfected). The expected value of the population of chronic cells is given by integrating with respect to the distribution $\overline{P_{2}}$, over all possible delay values, obtaining

$$
C(t)=\mathcal{E}_{2}[C(t, \tau)]=\int_{0}^{\infty} C(t ; \tau) d \bar{P}_{2}(\tau)
$$

Here the parameters $r_{v}, \delta_{C}, \delta$ and $\gamma$ are appropriate rate parameters (for details, see (Banks and Holte, 2003)). Therefore, the rate of change in the total population of chronic cells is governed by

$\dot{C}(t)=\mathcal{E}_{2}[\dot{C}(t ; \tau)]=\left(r_{v}-\delta_{C}-\delta X(t)\right) \mathcal{E}_{2}[C(t ; \tau)]+\gamma \int_{0}^{\infty} A(t-\tau) d \bar{P}_{2}(\tau)$

$C(0)=C_{0}$,

where $C_{0}$ is the initial condition for the total chronically infected cell population.

Next consider the delay between viral infection and viral production for the acutely infected cells $A(t)$. Again, it is unreasonable to expect the entire population of acutely infected cells to simultaneously commence viral production $\tau_{1}\left(\tau_{1}>0\right)$ hours after infection. Suppose that the delay between infection and production (for acutely infected cells $A(t)$ ) varies across the population with probability distribution $\overline{P_{1}}$ (again we do not assume absolute continuity of the associated measure). We also partition the expected total viral population $V$ into those virions $V_{A}$ produced by acutely infected cells and those virions $V_{C}$ produced by chronically infected cells so that

$V=V_{A}+V_{C}$.

Then we denote by $V_{A}(t ; \tau)$ the subpopulation of virus which are produced by an acutely infected cell $\tau$ hours after being infected. Thus, the rate of change in this subgroup of virions is governed by

$\dot{V}_{A}(t ; \tau)=-c V_{A}(t ; \tau)+n_{A} A(t-\tau)-n_{I} V_{A}(t ; \tau) T(t)$.

To obtain the (expected) number of virus at time $t$ that have been produced by acutely infected cells, we must integrate with respect to the distribution $\overline{P_{1}}$, over all possible delays

$V_{A}(t)=\mathcal{E}_{1}\left[V_{A}(t ; \tau)\right]=\int_{0}^{\infty} V_{A}(t ; \tau) d \bar{P}_{1}(\tau)$

which yields the governing equation for this larger subpopulation of virions 
$\dot{V}_{A}(t)=\mathcal{E}_{1}\left[\dot{V}_{A}(t ; \tau)\right]$

$=-c V_{A}(t)+n_{A} \int_{0}^{\infty} V_{A}(t ; \tau) d \bar{P}_{1}(\tau)-n_{I} V_{A}(t) T(t)$.

To account for the chronically infected cells as a source of virions, we denote by $V_{C}$ the subpopulation of virions produced by chronically infected cells. Thus the equation describing the rate of change in the size of this subpopulation is

$\dot{V}_{C}(t)=-c V_{C}(t)+n_{C} C(t)-n_{I} V_{C}(t) T(t)$,

where the expected value $C$ of the total population of chronically infected cells is defined in equation (2). Therefore, the governing equations for the total population of virus are described by

$\dot{V}(t)=\mathcal{E}_{1}\left[\dot{V}_{A}(t ; \tau)+\dot{V}_{C}(t)\right]$

$=-c\left(V_{A}(t)+V_{C}(t)\right)-n_{I}\left(V_{A}(t)+V_{C}(t)\right) T(t)+n_{C} C(t)+n_{A} \int_{0}^{\infty} A(t-\tau) d \bar{P}_{1}(\tau)$

$=-c V(t)+n_{A} \int_{0}^{\infty} A(t-\tau) d \bar{P}_{1}(\tau)+n_{C} C(t)-n_{I} V(t) T(t)$

$V(0)=V_{0}$,

where $V_{0}$ is the initial condition for the total virions population.

Moreover, we assume that the $A$ and $T$ subclasses have no subpopulation structures, and are therefore governed by

$\dot{A}(t)=\left(r_{v}-\delta_{A}-\delta X(t)\right) A(t)+n_{I} V(t) T(t)-\gamma \int_{0}^{\infty} A(t-\tau) d \bar{P}_{2}(\tau)$

$A(0)=A_{0}$

$\dot{T}(t)=\left(r_{u}-\delta_{u}-\delta X(t)-n_{I} V(t)\right) T(t)+S$

$T(0)=T_{0}$,

with initial conditions $A_{0}$ and $T_{0}$. Note that in equation (3), the rate term with the delay (representing the delayed conversion of $A$ to $C$ ) is simply the negative of the corresponding delay rate term in equation (3).

Finally, we make the change of variables $P_{i}(\xi)=\overline{P_{i}}(-\xi)$ so that the distributions are now defined on $(-\infty, 0)$ instead of $(0, \infty)$ (we do this to be consistent with the standard notation in the FDE literature), and obtain the system

$$
\begin{aligned}
& \dot{V}(t)=-c V(t)+n_{A} \int_{-\infty}^{0} A(t+\theta) d P_{1}(\theta)+n_{C} C(t)-n_{I} V(t) T(t) \\
& \dot{A}(t)=\left(r_{v}-\delta_{A}-\delta X(t)\right) A(t)+n_{I} V(t) T(t)-\gamma \int_{-\infty}^{0} A(t+\theta) d P_{2}(\theta) \\
& \dot{C}(t)=\left(r_{v}-\delta_{A}-\delta X(t)\right) C(t)+\gamma \int_{-\infty}^{0} A(t+\theta) d P_{2}(\theta) \\
& \dot{T}(t)=\left(r_{v}-\delta_{A}-\delta X(t)-n_{I} V(t)\right) T(t)+S
\end{aligned}
$$


which is a vector system of the form (1). Special cases of such systems include those in which the probability measures are defined on some finite interval $Q=[-r, 0]$ of possible delay values $\theta$ as in (1).

This model was successfully used (see (Banks and Holte, 2003)) to describe in vitro mice data from Dr. Michael Emerman's lab at Fred Hutchinson Cancer Research Center. Using inverse problem methodology and statistical analysis it was shown that improvement of fit to data by inclusion of the delays $\tau_{1}$ or $P_{1}$ is statistically significant while inclusion of delays $\tau_{2}$ is less important. Indeed, it was found that the experimental data could not be properly fit with an ODE version (i.e., with the delays omitted) of the model.

\section{EXAMPLE FROM A VACCINE PRODUCTION MODEL}

A second class of models (Banks and Allnutt, to appear) that illustrate the type of problems focused on here involve the use of shrimp grown in production "raceways" (essentially large growth chambers where environmental factors such as temperature, oxygen, nutrient levels, etc., can be carefully controlled) artificially infected to efficiently produce large quantities of an associated vaccine. Scientifically, this entails recruiting the biochemical machinery in an existing biomass for the production of a vaccine or antibody by infection using a virus carrying a passenger gene for the desired antibody response.

While the model of (Banks and Allnutt, to appear) is specific to virus growth and vaccine production in shrimp, the implications for other crustaceans are obvious. And of course the shrimp models we investigate can serve as a foundation for understanding viral progression in other species important to marine agriculture. The mathematical goal is to model a system wherein one uses shrimp as a scaffold organism to produce biological countermeasures. In such a system one might first stock shrimp postlarvae and allow them to grow normally in the controlled environment. Then one infects them with a recombinant viral vector (e.g., recombinant Taura Syndrome virus or rTSV in the example developed in (Banks and Allnutt, to appear)) expressing a foreign antigen, resulting in vaccine production in live infected shrimp.

To mathematically demonstrate the feasibility of this approach one considers a hybrid model of the shrimp biomass/countermeasure production system which has two components: biomass production, and production of countermeasure (antibody/vaccine). The output of the biomass production model is input to the vaccine production model. For initial investigations the amount of vaccine produced is assumed equal to the total infected biomass. Thus, the vaccine production model will essentially follow the course of the viral dynamics in the shrimp.

The effort requires modeling the dynamics of shrimp at the population level. In such models ignoring structure in constructing mathematical models for the dynamics of shrimp is unrealistic, since shrimp have size dependent characteristics as well as responses to external environment. An appropriate beginning model is based on the classical McKendrick-vonFoerster/Sinko-Streifer size-structured population equations (Kot, 2001; Metz and Diekmann, 1986) with mass as the structure variable, i.e., one equates the size variable with the mass in the model.

While there appears to be a dearth of literature on modeling epidemics in shrimp populations, in (Lotz and Breland, 2003) the authors develop a non-structured five compartment epidemic model of TSV that includes a Reed-Frost transmission process in closed populations of shrimp (Litopenaeus vannamei). However, structure can play an important role in the study of viral epidemiology in shrimp. Moreover, experimental results (Hasson and White, 1999) suggest that the mortality rate in acutely infected shrimp depends on the length of time that the shrimp remain acute. Also, individuals in the latent phase have varying residency times before they progress into the acute phase. To incorporate all of these features, the authors of (Banks and 
Allnutt, to appear) attempted to model the progression of TSV in shrimp in a system of delay PDEs. However, it is difficult to correctly account for the different residency periods of individual shrimp in this fashion as the size of the shrimp is a function of time. Instead of tracing back in time to incorporate delays, a different approach involves recording the variable residency times in the different stages by introducing a new variable which one calls the class age of an individual. The class age of an individual in a given stage represents the length of time that the individual spends in that stage and serves as a surrogate for time delays. A similar approach has been used to investigate a linear cell population model. In such models, cells are assumed capable of simultaneous proliferation and maturation where in the proliferating phase, cells are committed to undergo cell division some time units after entering this phase (Adimy and Pujo-Menjouet, 2003).

In the vaccine production stage the shrimp are infected by distributing chopped dead shrimp infected with a recombinant virus evenly throughout the raceway. This transfected biomass is sufficiently large so that most of the shrimp can be infected in a short period of time, such as one day. There are other modes of transmission of virus in shrimp, such as cohabitation with infected shrimp that may be shedding the virus into the surrounding medium (waterborne infection). However, compared to the probability of shrimp becoming infected via ingestion, these modes of transmission can be assumed (reasonably for a first investigation) to be negligible. Hence one might only assume infection via ingestion of dead transfected biomass. It might be further assumed that all the shrimp have an equal chance of becoming infected by eating the infected biomass. A reasonable time interval for infection is 7 to 10 days. From (Lotz and Breland, 2003) and (Dhar and Walker, 2004) one knows that during this time interval almost no shrimp progress into the chronic state. Therefore it is reasonable to consider the following three compartment states: susceptible $(S)$, latently infected $(L)$ and acutely infected (A) in a model. In this model, it is assumed that shrimp will become instantly infected (i.e., progress into latent state) as soon as they ingest some of the infected biomass. As we have noted earlier, however, experimental observations suggest that there exists a temporal delay between the initial latent infection and initial acute infection (Hasson and White, 1999). Moreover, it is biologically unrealistic to expect all members of the shrimp population to progress into the acute phase at a fixed number of days after initial latent infection. In addition the shrimp in the acute phase have varying mortality rates because of the different times that they progress into the acute phase and also due to the differences in genetic make-up of the host. As we have already noted, it is difficult to account for the class age history (i.e., the length of time that shrimp spend in a state) of shrimp in a particular (latent or acute) state using a system of delay PDE's with only size as the structure variable. This is because it is not obvious how to correctly represent the integral involving the delay. As an alternative, in order to model variable residency times one may keep track of the class age and the size of shrimp by incorporating both size structure and class age structure into the latent and acute states.

Based on experimental findings, it is reasonable to assume that there is a positive probability that shrimp can stay in each the latent and acute state for more than 7 to 10 days. Thus one can assume that the class age interval for both states is the same as the time interval $T_{V}$ that we consider in our model. Note that all shrimp from the biomass production raceway are healthy; there are no latently infected or acutely infected shrimp in the raceway at time $t=0$. We also know that shrimp in the acute state stop growing, which means that the growth rate in this state is $g=0$.

Based on the above discussions, the vaccine production model developed in (Banks and Allnutt, to appear) is given by 


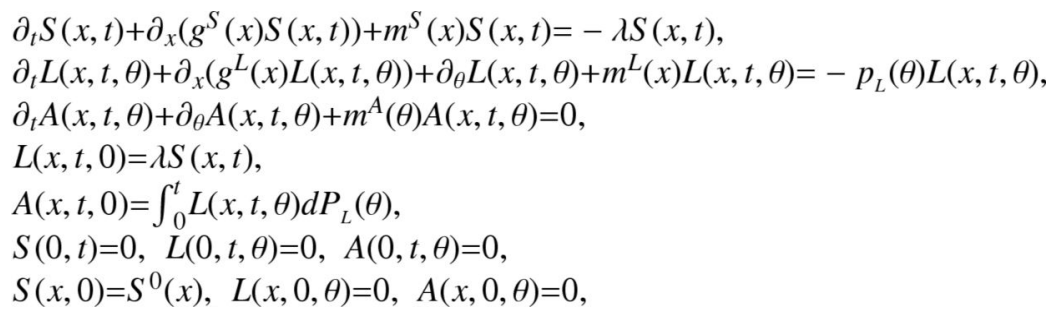

where $(x, t, \theta) \in\left[x_{\min }, x_{\max }\right] \times\left[0, T_{V}\right] \times\left[0, T_{V}\right]$. In the above $S(x, t)$ denotes the density of individuals having mass $x$ at time $t$ and $\partial_{t}=\frac{\partial}{\partial t}$. The function $L(x, t, \theta)$ denotes the density of individuals having mass $x$ at time $t$ that have spent $\theta$ days in the latent state, whereas the function $A(x, t, \theta)$ denotes the density of individuals having mass $x$ at time $t$ that have spent $\theta$ days in the acute state. The quantity $g^{S}(x)$ denotes the growth rate of individuals in the susceptible state, $g^{L}(x)$ denotes the growth rate of individuals in the latent state. The function $m^{S}(x)$ denotes the mortality rate of individuals in the susceptible state, and the function $m^{L}(x)$ denotes the mortality rate of individuals in the latent state, and $m^{A}(\theta)$ denotes the mortality rate of the shrimp that spend $\theta$ days in the acute state. The latent to acute probability density rate function $p_{L}(\theta)=P_{L}^{\prime}(\theta)$ defined for $\theta \in\left[0, T_{V}\right]$ denotes the rate at which the shrimp in the latent state that have spent $\theta$ days in the latent state become acutely infected, while the quantity $\lambda$ denotes the infection rate due to ingestion of chopped infected shrimp. Finally $S^{0}(x)$ denotes the initial population density of susceptible shrimp produced from the biomass production model.

We note that this is again a probability distribution $\left(P_{L}\right)$ dependent dynamical system (in this case a complicated system of partial differential equations) for which the distribution $P_{L}$ must be estimated in some type of inverse problem.

\section{PROBLEM FORMULATION FOR DISTRIBUTION DEPENDENT DYNAMICS}

In both the examples cited above as well as in many others, a major effort involves estimation of the probability distributions $P$ from data. A typical inverse problem consists of minimizing the output least squares criterion

$$
J(P)=\sum_{i=1}^{n}\left|x\left(t_{i} ; P\right)-\widehat{d_{i}}\right|^{2}
$$

where $\left\{\hat{d}_{i}\right\}$ is the data and $x(t ; P)$ represents the solution to the distribution dependent dynamics such as (1), (3), or (4). The minimization is to be carried out over the space $\mathcal{P}(Q)$ of probability measures defined on a set $Q$ of possible parameter values. For example, in the systems presented above, the delay times or residency times are restricted to some finite interval $Q=[-r, 0]$ or $Q=\left[0, T_{V}\right]$, respectively. In addition to such inverse or estimation problems, we are also concerned with other questions for problems that have distribution dependent dynamics including the existence and uniqueness of solutions to the dynamical system, continuous dependence of solutions, sensitivity of solutions with respect to the probability distributions, and numerical approximations. In order to deal with such questions (either theoretical or computational) one needs a topology on the measure space $\mathcal{P}(Q)$. Indeed, one needs a number of items to develop theoretical and computational foundations including

i. A topology on $\mathcal{P}(Q)$, 
ii. Continuity of $P \rightarrow J(P)$ in this topology,

iii. Compatible compactness results (for well-posedness),

iv. Approximations in this topology (for computations).

It is fortunate that probability theory offers significant conceptual help toward a possible complete, tractable computational methodology (Billingsley, 1968). The primary tool is the Prohorov metric, which can be formally defined as follows. Suppose $(Q, d)$ is a complete metric space. For any closed subset $F \subset Q$ and $\varepsilon>0$, define

$F^{\mathcal{E}}=\{q \in Q: d(\tilde{q}, q)<\varepsilon, \tilde{q} \in F\}$.

Define the Prohorov metric $\rho: \mathcal{P}(Q) \times \mathcal{P}(Q) \rightarrow \mathbb{R}^{+}$by

$\rho\left(P_{1}, P_{2}\right) \equiv \inf \left\{\varepsilon>0: P_{1}[F] \leq P_{2}\left[F^{\varepsilon}\right]+\varepsilon, F\right.$ closed, $\left.F \subset Q\right\}$.

This can be shown to be a metric on $\mathcal{P}(Q)$ that satisfies

a. $(\mathcal{P}(Q), \rho)$ is a complete metric space,

b. If $Q$ is compact, then $(\mathcal{P}(Q), \rho)$ is a compact metric space.

We note that the definition of $\rho$ is not intuitive. It is not clear, for example, what " $P_{k} \rightarrow P$ " in $\rho$ metric means. One finds the following important characterization (Billingsley, 1968).

\section{Theorem 1}

Given $P_{k}, P \in \mathcal{P}(Q)$, the following convergence statements are equivalent:

i. $\rho\left(P_{k}, P\right) \rightarrow 0$,

ii. $\int_{Q} f d P_{k}(q) \rightarrow \int_{Q} f d P(q)$ for all bounded, uniformly continuous $f: Q \rightarrow \mathbb{R}^{1}$,

iii. $P_{k}[A] \rightarrow P[A]$ for all Borel sets $A \subset Q$ with $P[\partial A]=0$ :

Additional useful results include:

- $\quad$ Let $C_{B}^{*}(Q)$ denote the topological dual of $C_{B}(Q)$, where $C_{B}(Q)$ is the usual space of bounded continuous functions on $Q$ with the supremum norm. If we view $\mathcal{P}(Q) \subset C_{B}^{*}(Q)$, convergence in the $\rho$ topology is equivalent to weak* convergence in $\mathcal{P}(Q)$,

- Convergence in the $\rho$ metric is equivalent to convergence in distribution.

Considering (ii) of Theorem 1, it is readily argued that the dynamics of systems such as (1) (and (3), (4)) are $\rho$-continuous in $P$ on $\mathcal{P}(Q)$. Standard arguments from the theory of differential equations can then be used to argue that the mapping $P \rightarrow x(t ; P)$ is also continuous on $(\mathcal{P}(Q)$, $\rho$ ). This yields immediately that

$P \rightarrow J(P)=\sum_{i=1}^{n}\left|x\left(t_{i} ; P\right)-\widehat{d_{i}}\right|^{2}$ 
is continuous in the $\rho$ topology. Continuity of $P \rightarrow J(P)$ and compactness of $\mathcal{P}(Q)$ (each with respect to the $\rho$ metric) allows one to assert the existence of a solution to min $J(P)$ over $P \in \mathcal{P}$ $(Q)$.

As we shall see below, the Prohorov metric is also fundamental to development of a sensitivity theory as well as "finite element" type approximation schemes for the systems of interest here.

4.1 Abstract Formulation-Before we present some theoretical results on a class of distribution dependent problems, we illustrate how to derive an abstract Cauchy formulation in a complex Banach space for a typical delay system example such the HIV model above. First, we assume the HIV system derived in Section 2 only depends on absolutely continuous (continuous) distributions; that is, $d P_{i}(\tau)=p_{i}(\tau) d \tau$ for $i=1,2$ where $p_{i}(\tau)$ are probability densities. A more general framework for discrete distributions and mixed (a combination of continuous and discrete) distributions is discussed in the next section where one assumes the form

$$
P(\tau)=\sum_{i=1}^{k} p_{i} \Delta_{\tau_{i}}(\tau)
$$

and

$$
P(\tau)=\sum_{i=1}^{k} p_{i} \Delta_{\tau_{i}}(\tau)+\int_{-r}^{\tau} p(\xi) d \xi
$$

Here $\Delta_{\tau}$ is the Dirac measure with atom (mass) at $\tau$ for the discrete and mixed measures, respectively.

Returning to the HIV model we let $v=(V, A, C, T)^{T}$ and $x(t)=\left(v(t), v_{t}\right) \in X=\mathbb{R}^{4} \times L^{2}(-r, 0$; $\left.\mathbb{R}^{4}\right)$. For $0<r<\infty$, we denote the parameter space $\boldsymbol{m}_{\boldsymbol{C}}=L^{2}(-r, 0) \times L^{2}(-r, 0)$ and $\boldsymbol{m}_{C}=\left\{\left(p_{1}\right.\right.$, $\left.p_{2}\right) \in \mathscr{m} \mid p_{1}, p_{2} \geq 0$ and $\left.\int_{-r}^{0} p_{i}(\tau) d \tau=1\right\}$. Then the HIV system derived in equation (3) can be rewritten as an abstract Cauchy problem

$$
\begin{aligned}
& \dot{x}(t)=\mathcal{A} x(t)+f_{2}(t), \quad t \geq 0 \\
& x(0)=x_{0},
\end{aligned}
$$

where $f_{2}(t)=\left((0,0,0, S)^{T}, 0\right) \in X$, and $x_{0}=(\eta, \varphi) \in X$. Here $\mathcal{A}$ is a nonlinear operator such that $\mathcal{A}: \mathcal{D}(\mathcal{A}) \subset X \rightarrow X$ and $\mathcal{A}(\eta, \varphi)=\left(L(\eta, \varphi)+f_{1}(\eta), \frac{d}{d \tau} \varphi\right)$ where $\mathcal{D}(\mathcal{A})=\{(\eta, \varphi) \in X \mid \varphi \in$ $H^{1}\left(-r, 0 ; \mathbb{R}^{4}\right)$ and $\left.\eta=\varphi(0)\right\}$. Furthermore, for $\left((\eta, \varphi) \in \mathbb{R}^{4} \times L^{2}\left(-r, 0 ; \mathbb{R}^{4}\right)\right.$, 


$$
\begin{aligned}
& L(\eta, \varphi)=\left[\begin{array}{cccc}
-c & 0 & n_{c} & 0 \\
0 & r_{v}-\delta_{A} & 0 & 0 \\
0 & 0 & r_{v}-\delta_{c} & 0 \\
0 & 0 & 0 & r_{u}-\delta_{u}
\end{array}\right] \eta \\
& +n_{A}\left[\delta_{(1,2)}\right]_{(4,4)} \int_{-r}^{0} \varphi(\tau) p_{1}(\tau) d \tau \\
& +\gamma\left(\left[\delta_{(3,2)}\right]_{(4,4)}-\left[\delta_{(2,2)}\right]_{(4,4)}\right) \int_{-r}^{0} \varphi(\tau) p_{2}(\tau) d \tau \\
& f_{1}(\eta)=\left[\begin{array}{c}
-\alpha \eta_{1} \eta_{4} \\
-\delta\left(\sum_{i=2}^{4} \eta_{i}\right) \eta_{2}+\alpha \eta_{1} \eta_{4} \\
-\delta\left(\sum_{i=2}^{4} \eta_{i}\right) \eta_{3} \\
-\delta\left(\sum_{i=2}^{4} \eta_{i}\right) \eta_{4}-\alpha \eta_{1} \eta_{4}
\end{array}\right],
\end{aligned}
$$

where $\left[\delta_{(i, j)}\right]_{(4 ; 4)}$ is a $4 \times 4$ matrix with a one in the $(i, j)^{\text {th }}$ component and zeros everywhere else. In (Banks and Bortz, 2005a), (Banks and Holte, 2003) the mass action product nonlinearities in $f_{1}$ are replaced by saturating nonlinear functions - see the definition of $\bar{f}_{1}$ in (Banks and Bortz, 2005a), (Banks and Holte, 2003).

Once an abstract Cauchy formulation is constructed, existence and uniqueness of solutions for equation (8) follow from the results in (Banks and Bortz, 2005b; Banks and Nguyen, to appear) which are summarized in Section 4.2 below. Moreover, theoretical results in (Banks and Nguyen, to appear) also provide continuous dependence of solutions along with the derivation of the sensitivity function for general nonlinear ordinary differential equations (ODEs) in a Banach space. Here we only show the construction of the abstract Cauchy problem for delay systems with continuous probability measures. However, an abstract Cauchy formulation for delay systems with discrete and mixed distributions of the form (6) and (7), respectively, can also be constructed using similar concepts but with different parameter spaces. These theoretical results and associated parameter spaces of probability measures are the focus of the next section.

4.2 Theoretical Results-In this section we recall theoretical results that treat delay systems with absolutely continuous (continuous) distributions. Interested readers can find more details on the theories and the proofs in (Banks and Nguyen, to appear). As one can see from the previous section, time delay problems with absolutely continuous (continuous) distributions are a special case of an abstract nonlinear ODE where the state space is a general Banach space $\mathrm{X}$ and the parameter space $\oiint_{c}$ is a convex subset of a Banach space $\boldsymbol{m}_{\text {such }}$ as $\boldsymbol{m}_{C}=L^{2} \times L^{2}$. Therefore, consider a general nonlinear ODE of the form

$$
\dot{x}(t)=f(t, x(t), \mu),
$$

where $f: \mathbb{R}_{+} \times X \times \not{m} \rightarrow X$ and $X$ and $\mathscr{m}$ are complex Banach spaces. We define the successive approximations for system (9) to be the functions, $x^{0}, x^{1}, \ldots$, given recursively by

$x^{0}\left(t, t_{0}, x_{0}, \mu\right)=x_{0}$,

$x^{k+1}\left(t, t_{0}, x_{0}, \mu\right)=x_{0}+\int_{t_{0}}^{t} f\left(s, x^{k}\left(s, t_{0}, x_{0}, \mu\right), \mu\right) d s$, 
for $k=0,1,2, \ldots$ Then one can establish the following theoretical results.

\section{Lemma 1}

(Existence and Uniqueness of Solutions) Let $\mathrm{f}: \mathbb{R}_{+} \times \mathrm{X} \times \boldsymbol{M} \rightarrow \mathrm{X}$ be continuous and

$\left|f\left(t, x_{1}, \mu\right)-f\left(t, x_{2}, \mu\right)\right| \leq C\left|x_{1}-x_{2}\right|$

for some constant $C>0$. Then the successive approximations $x^{k}$ converge uniformly for $t \in$ $\left[t_{0}, T\right]$ to a unique solution $x$ of (9) such that $x\left(t_{0}, t_{0}, x_{0}, \mu\right)=x_{0}$.

\section{Lemma 2}

(Continuous Dependence of Solutions on Parameters) Let $f \in C\left[\mathbb{R}_{+} \times X \times \infty, X\right]$ and for $\mu=$ $\mu_{0}$, let $x\left(t, t_{0}, x_{0}, \mu_{0}\right)$ be the solution of

$\dot{x}=f\left(t, x, \mu_{0}\right), \quad x\left(t_{0}\right)=x_{0}$,

existing on $\left[t_{0}, T\right]$. Assume further that

$\lim _{\mu \rightarrow \mu_{0}} f(t, x, \mu)=f\left(t, x, \mu_{0}\right)$,

uniformly in $(t, x)$ and for $\left(t, x_{1}, \mu\right) ;\left(t, x_{2}, \mu\right) \in \mathbb{R}_{+} \times X \times \not$,

$\left|f\left(t, x_{1}, \mu\right)-f\left(t, x_{2}, \mu\right)\right| \leq C\left|x_{1}-x_{2}\right|$

for some constant $C>0$. Then the differential system

$\dot{x}=f(t, x, \mu), \quad x\left(t_{0}\right)=x_{0}$,

has a unique solution $x\left(t, t_{0,}, x_{0}, \mu\right)$ satisfying

$\lim _{\mu \rightarrow \mu_{0}} x\left(t, t_{0}, x_{0}, \mu\right)=x\left(t, t_{0}, x_{0}, \mu_{0}\right), \quad t \in\left[t_{0}, T\right]$.

Even though the results given here are under the assumed global Lipschitz condition, similar results can also be established under the weaker assumptions of a local Lipschitz condition and $f$ being dominated by an affine function. We let $B(X, Y)$ denote the space of bounded linear operators from $X$ onto $Y$ and summarize a sensitivity theory for delay systems with absolutely continuous (continuous) measures in Theorems 2 and 3.

\section{Theorem 2}

Suppose the function $f(t, x, \mu)$ of $(9)$ has continuous Frechet derivatives $f_{x}(t, x, \mu)$ with respect to $\mathrm{x}$ and $\mathrm{f}_{\mu}(\mathrm{t}, \mathrm{x}, \mu)$ with respect to $\mu$ with 
$\left|f_{x}(t, x, \mu)\right| \leq M_{0} \quad$ and $\quad\left|f_{\mu}(t, x, \mu)\right| \leq M_{1}$

for some constants $M_{0}>0$ and $M_{1}>0$. Then the Frechet derivative $y(t)=\frac{\partial}{\partial \mu} x\left(t, t_{0}, x_{0}, \mu\right)_{\text {exists }}$ with $y(t)$ in $B(\not D, X)$ satisfying the equation

$\dot{y}(t)=f_{x}\left(t, x\left(t, t_{0}, x_{0}, \mu\right), \mu\right) y(t)+f_{\mu}\left(t, x\left(t, t_{0}, x_{0}, \mu\right), \mu\right)$,

$y\left(t_{0}\right)=0$,

for $t \geq t_{0}$.

With the parameter space of probability density functions $\mathbb{M}_{c}$, which is a convex subset of a Banach space $\%$, the sensitivity theory (Theorem 2) above can also be applied using directional derivatives instead of the Frechet derivative. However, it is shown in (Banks and Nguyen, to appear) that the directional derivative of a continuous function $g$ is the Frechet derivative on

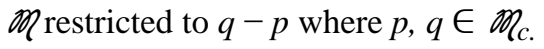

In order to accommodate delay problems with Dirac (discrete) measures or measures with a continuous component and a saltus component, the theoretical results above are extended to a general convex metric space. This is necessary because the parameter space associated with the Prohorov metric is no longer a Banach space but only special case of a convex metric space $\left(m_{1}, d_{m_{1}}\right)$. For discrete measure delay systems where the measures are defined in $(6)$, the parameter space $\left(\mathscr{D}_{1}, d_{m_{1}}\right)$ is normally chosen to be a topology with the Prohorov metric $(\mathcal{P}$ $(Q), \rho)$. Although the Prohorov metric is not conceptually easy to use, it generates a similar topology to the weak $L^{2}$ topology (e.g., see (Banks and Pinter, 2005)) which is of course the same as the weak* topology in this case. Therefore, the Prohorov metric may be applied in numerical approximation in distribution dependent problems taking advantage of its relation in convergence to the weak $L^{2}$ convergence. For delay systems with mixed measures as defined in (7), the parameter space can be based on a combination of the Prohorov metric topology and the weak $L^{2}$ topology for compatibility. Therefore, Banks, Dediu and Nguyen have extended the theoretical results mentioned above to the case where the parameter space is a convex metric space. Let $\left(\mathscr{m}_{1}, d_{m_{1}}\right)$ denote a general convex metric space with distance $d_{m_{1}}$ and $X$ denote a general complex Banach space. Consider a general nonlinear abstract ODE

$$
\dot{x}(t)=f\left(t, x(t), \mu_{1}\right), x(0)=0,
$$

where $f: \mathbb{R}_{+} \times X \times \mathbb{m}_{1} \rightarrow X$ is continuous in all three variables and Frechet differentiable in $x$. Here the solution $x \in X$ and the parameter $\mu_{1} \in m_{1}$. The conditions for and statement of existence and uniqueness of solutions of equation (10) along with continuous dependence of solutions for the general convex metric parameter space are similar to those for the situation detailed above where the parameter space is a general complex Banach space; therefore, those theoretical results are not repeated here. When deriving the sensitivity theory for the convex metric parameter space case, the directional derivative is used instead of the Frechet derivative with respect to the measures.

Given any two arbitrary points $\mu_{1}, v \in\left(m_{1}, d_{m_{1}}\right)$, we define the directional derivative $\delta f(t, x$, $\left.\mu_{1} ; v-\mu_{1}\right)$ of $f$ at $\mu_{1}$ in the direction $v-\mu_{1}$ to be the value of the limit 


$$
\lim _{\substack{\varepsilon \rightarrow 0 \\ \varepsilon>0}} \frac{f\left(t, x, \mu_{1}+\varepsilon\left(v-\mu_{1}\right)\right)-f\left(t, x, \mu_{1}\right)}{\varepsilon}=\delta f\left(t, x, \mu_{1} ; v-\mu_{1}\right),
$$

provided this limit exists in $X$. A sensitivity theory for a convex metric parameter space is stated next; more details and proofs can be found in (Banks and Nguyen, 2006).

\section{Theorem 3}

Suppose the function $f\left(t, x, \mu_{1}\right)$ of $(10)$ has a Frechet derivative $f_{x}\left(t, x, \mu_{1}\right)$ with respect to $x$ such that $f_{\mathrm{X}} \in C\left[\mathbb{R}_{+} \times X \times \oiint_{1}, B(X, X)\right]$ and $\left|f_{x}\left(t, x, \mu_{1}\right)\right| \leq M_{0}$ for some constant $M_{0}>0$. Moreover, assume falso has a continuous directional derivative $\delta f\left(t, x, \mu_{1} ; v-\mu_{1}\right)$ with respect to $\mu_{1}$ in the direction of $\left(v-\mu_{1}\right)$ such that $\left|\delta f\left(t, x, \mu_{1} ; v-\mu_{1}\right)\right| \leq M_{1}$ where $M_{1}>0$. Then the directional derivative $y(t)=\delta x\left(t, \mu_{1} ; v-\mu_{1}\right)$ exists, with $y: \mathbb{R}_{+} \times X \times \oiint_{1} \rightarrow X$, and y satisfies the equation

$\dot{y}(t)=f_{x}\left(t, x\left(t, t_{0}, x_{0}, \mu_{1}\right), \mu_{1}\right) y(t)+\delta f\left(t, x\left(t, t_{0}, x_{0}, \mu_{1}\right), \mu_{1} ; \gamma-\mu_{1}\right)$, $y\left(t_{0}\right)=0$.

Having presented theoretical results to deal with delay differential equations where the time delay is distributed with different types of probability measures (i.e, absolutely continuous, continuous, discrete and mixed measures), we next discuss some numerical approximation issues for this class of problems.

4.3 Approximation Issues-We first note that even when the parameter set $Q$ is finite dimensional, the metric space $(\mathcal{P}(Q), \rho)$ is infinite-dimensional and hence one must use finitedimensional approximations to obtain tractable computational algorithms. To this end, one may prove (see (Banks and Bihari, 2001))

\section{Theorem 4}

Let $Q$ be a complete, separable metric space with metric $d, \mathbb{B}$ the class of all Borel subsets of $Q$ and $\mathcal{P}(Q)$ the space of probability measures on $(Q, \mathbb{B})$. Let $Q_{0}=\left\{q_{j}\right\}_{j=1}^{\infty}$ be a countable, dense subset of $Q$. Then the set of $P \in \mathcal{P}(Q)$ such that $P$ has finite support in $Q_{0}$ and rational masses is dense in $\mathcal{P}(Q)$ in the $\rho$ metric. That is,

$\mathcal{P}_{0}(Q) \equiv\left\{P \in \mathcal{P}(Q): P=\sum_{j=1}^{k} p_{j} \delta_{q_{j}}, k \in \mathcal{N}^{+}, q_{j} \in Q_{0}, p_{j}\right.$ rational, $\left.\sum_{j=1}^{k} p_{j}=1\right\}$

is dense in $(\mathcal{P}(Q), \rho)$, where $\delta_{q j}$ is the Dirac measure with atom at $q_{j}$.

It is straight forward to use the ideas and results associated with this theorem to develop computationally efficient schemes. Given $Q_{d}=\bigcup_{M=1}^{\infty} Q_{M}$ with $Q_{M}=\left\{q_{j}^{M}\right\}_{j=1}^{M}$ (a "partition" of $Q$ ) chosen so that $Q_{d}$ is dense in $Q$, define 
$\mathcal{P}^{M}(Q)=\left\{P \in \mathcal{P}(Q): P=\sum_{j=1}^{M} p_{j} \delta_{q_{j}^{M}}, q_{j}^{M} \in Q_{M}, p_{j}\right.$ rational, $\left.\sum_{j=1}^{k} p_{j}=1\right\}$

Then we find

i. $\mathcal{P}^{M}(Q)$ is a compact subset of $(P(Q), \rho)$,

ii. $\mathcal{P}^{M}(Q) \subset \mathcal{P}^{M+1}(Q)$,

iii. " $\mathcal{P} M(Q) \rightarrow \mathcal{P}(Q)$ " in the $\rho$ topology; that is, elements in $\mathcal{P}(Q)$ can be approximated in the $\rho$ metric by elements of $P^{M}$ for $M$ sufficiently large.

These ideas and results can then be used to establish a type of "stability" of the inverse problem (see (Banks and Bihari, 2001), (Banks and Kunisch, 1989)). We first define a series of approximate problems consisting of minimizing

$J\left(P_{M}\right)=\sum_{i=1}^{n}\left|x\left(t_{i} ; P_{M}\right)-\widehat{d}_{i}\right|^{2}$

over $P_{M} \in \mathcal{P}^{M}(Q)$. Then we have

\section{Theorem 5}

Let $Q$ be a compact metric space and assume solutions $x(t, P)$ are continuous in $P$ on $(\mathcal{P}(Q)$, $\rho)$. Let $Q_{d}$ be a countable dense subset of $Q$ with $Q_{M}=\left\{q_{j}^{M}\right\}_{j=1}^{M}$ and $\mathcal{P}^{M}(Q)$ as above so that (i)(iii) holds. suppose $P_{M}^{*}\left(\widehat{d}^{k}\right)$ is the set of minimizers for $J\left(P_{M}\right)$ over $P_{M} \in \mathcal{P}^{M}(Q)$ corresponding to the data $\left\{\hat{d}^{k}\right\}$ and $P^{*}(\hat{d})$ is the set of minimizers over $P \in \mathcal{P}(Q)$ corresponding to $\hat{d}$, where $\hat{d}^{k}, \hat{d}^{k} \in \mathbb{R}^{n}$ are the observed data such that $\hat{d}^{k} \rightarrow \hat{d}$. Then dist $\left(P_{M}^{*}\left(\widehat{d}^{k}\right), P^{*}(\hat{d})\right) \rightarrow 0$ as $M \rightarrow$ $\infty$ and $\hat{d}^{k} \rightarrow \hat{d}$. Thus, the solutions depend continuously on the data and the approximate problems are method stable as formulated in (Banks and Kunisch, 1989).

Of course, for infinite dimensional state systems such as (1), (3) and (4), one would also approximate the solutions $x\left(t, P_{M}\right)$ by finite dimensional approximate solutions $x^{N}\left(t ; P_{M}\right)$ to obtain a completely finite dimensional problem. A version of the above theorem can be given for this simultaneous state/parameter approximation using the approach for state/parameter approximation found in (Banks and Kunisch, 1989).

The "delta measure" approximations given above are essentially zero-order splines. As one might expect, higher order schemes can readily be developed. An example of linear spline schemes has been developed in (Banks and Pinter, 2005) and further investigated in (Banks and Davis, to appear) and can be summarized in the following theorem.

\section{Theorem 6}

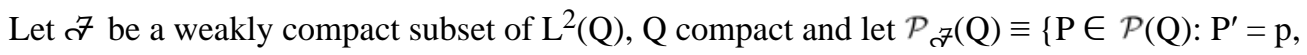
$\left.\mathrm{p} \in{ }^{7}\right\}$. Then $\mathcal{P}_{\mathcal{\gamma}}(\mathrm{Q})$ is compact in $(\mathcal{P}(\mathrm{Q}), \rho)$. Moreover, if we define $\left\{l_{j}^{M}\right\}$ to be the linear splines on $\mathrm{Q}$ corresponding to the partition $\mathrm{Q}_{\mathrm{M}}$, where ${ }_{M} Q_{M}$ is dense $Q$, and define 
$\mathcal{P}^{M} \equiv\left\{p^{M}: p^{M}=\sum_{j} b_{j}^{M} l_{j}^{M}, b_{j}^{M}\right.$ rationasl $\}$

Then if

$\mathcal{P}_{\mathscr{F} M} \equiv\left\{P_{M} \in \mathcal{P}(Q): P_{M}=\int p^{M}, p^{M} \in \mathcal{P}^{M}\right\}$,

we have $\cup_{M} \mathcal{P}_{\mathscr{F}}$ mis dense in $\left(\mathcal{P}_{\mathcal{7}}(Q), \rho\right)$.

\section{CONCLUDING REMARKS}

The framework outlined above, while most useful, is not complete. Statistical aspects of the inverse problems for estimation of measures as discussed above are still under investigation. The approximations (delta and splines) lead to finite dimensional inverse problems for which standard asymptotic theory (see Chapter 12 of (Seber and Wild, 1989)) for standard errors and confidence intervals (using the sensitivity functions discussed above) can be applied. However, an analogous asymptotic theory for the original infinite dimensional problems involving (5) of Section 4 has yet to be rigorously developed.

\section{Acknowledgments}

This research was supported in part by the Joint DMS/NIGMS Initiative to Support Research in the Area of Mathematical Biology under grant 1R01GM67299-01, in part by the U.S. Air Force Office of Scientific Research under grant AFOSR-FA9550-04-1-0220 and in part from support at the Statistical and Applied Mathematical Sciences Institute, which is funded by NSF under grant DMS-0112069.

\section{References}

Adimy M, Pujo-Menjouet L. A mathematical model describing cellular division with a proliferating phase duration depending on the maturity of cells. Elect J Diff Equations 2003;2003:1-14.

Banks HT, Bihari KL. Modeling and estimating uncertainty in parameter estimation. Inverse Problems 2001;17:1-17.

Banks HT, Bokil VA, Hu S, Dhar AK, Bullis RA, Browdy CL, Allnutt FCT. Shrimp biomass and viral infection for production of biological countermeasures. Math Biosci and Engr. December;2005 (to appear)CRSC-TR05-45

Banks HT, Bortz DM. A parameter sensitivity methodology in the context of HIV delay equation models. CRSC-TR02-24, August 2002; J Math Biol 2005a;50:607-625. [PubMed: 15614552]

Banks HT, Bortz DM. Inverse problems for a class of measure dependent dynamical systems. CRSCTR04-33, September, 2004; J Inverse and Ill-posed Problems 2005b;13:103-121.

Banks HT, Bortz DM, Holte SE. Incorporation of variability into the modeling of viral delays in HIV infection dynamics. CRSC-TR01-25, November, 2001; Math Biosci 2003;183:63-91. [PubMed: 12604136]

Banks, HT.; Bortz, DM.; Pinter, GA.; Potter, LK. Modeling and imaging techniques with potential for application in bioterrorism. In: Banks, HT.; Castillo-Chavez, C., editors. Frontiers in Applied Math, Bioterrorism: Mathematical Modeling Applications in Homeland Security. Vol. FR28. SIAM; Philadelphia, PA: 2003. p. 129-154.

Banks HT, Davis JL. A comparison of approximation methods for the estimation of probability distributions on parameters. Applied Numerical Mathematics. October;2005 (to appear)CRSCTR05-38 
Banks HT, Dediu S, Nguyen HK. Sensitivity of dynamical systems to parameters in a convex subset of a topological vector space. 2006 September;CRSC-TR06-25

Banks HT, Gibson NL. Well-posedness of solutions with a distribution of dielectric parameters. Applied Mathematics Letters 2005;18:423-430.

Banks HT, Gibson NL. Electromagnetic inverse problems involving distributions of dielectric mechanisms and parameters. Quarterly of Applied Mathematics August;2005 CRSC-TR05-29(to appear)

Banks HT, Hood JB, Medhin NG. A molecular based model for polymer viscoelas-ticity: Intra- and intermolecular variability. Applied Mathematical Modelling. December;2004 (submitted)CRSCTR04-39

Banks, HT.; Kunisch, K. Estimation Techniques for Distributed Parameter Systems. Birkhauser; Boston: 1989.

Banks HT, Kurdila AJ, Webb G. Identification of hysteretic control influence operators representing smart actuators, Part I: Formulation. Mathematical Problems in Engineering 1997a;3:287-328.

Banks HT, Kurdila AJ, Webb G. Identification of hysteretic control influence operators representing smart actuators, Part II. Convergent approximations. J of Intelligent Material Systems and Structures 1997b;8:536-550.

Banks HT, Medhin NG, Pinter GA. Nonlinear reptation in molecular based hysteresis models for polymers. CRSC-TR03-45, December, 2003; Quarterly Applied Math 2004;62:767-779.

Banks HT, Medhin NG, Pinter GA. Multiscale considerations in modeling of nonlinear elastomers. J Comp Meth Sci and Engr. October;2003 (to appear)CRSC-TR03-42

Banks HT, Medhin NG, Pinter GA. Modeling of viscoelastic shear: A nonlinear stick-slip formulation. Differential Equations and Nonlinear Mechanics. February;2006 (submitted)CRSC-TR06-07

Banks HT, Nguyen HK. Sensitivity of dynamical systems to Banach space parameters. J Math Analysis and Applications. February;2005 (to appear)CRSC-TR05-13

Banks HT, Pinter GA. A probabilistic multiscale approach to hysteresis in shear wave propagation in biotissue. CRSC-TR04-03, January, 2004; SIAM J Multiscale Modeling and Simulation 2005;3:395412 .

Billingsley, P. Convergence of Probability Measures. Wiley; New York: 1968.

Dhar AK, Cowley JA, Hasson KW, Walker PJ. Genomic organization, biology, and diagnosis of Taura Syndrome Virus and Yellohead Virus of Penaeid Shrimp. Advances in Virus Research 2004;63:353420. [PubMed: 15530565]

Hasson KW, Lightner DV, Mohney LL, Redman RM, Poulos BT, White BM. Taura Syndrome Virus (TSV) lesion development and the disease cycle in the Pacific white shrimp Penaeus vannamei. Diseases of Aquatic Organisms 1999;36:81-93.

Lotz JM, Flowers AM, Breland V. A model of Taura Syndrome Virus (TSV) epidemics in Litopenaeus Vannamei. J Inver Path 2003;83:168-176.

Kot, M. Elements of Mathematical Ecology. Cambridge University Press; Cambridge: 2001.

Metz, JAJ.; Diekmann, EO. Lecture Notes in Biomathematics. Vol. 68. Springer; Heidelberg: 1986. The Dynamics of Physiologically Structured Populations.

Seber, GAF.; Wild, CJ. Nonlinear Regression. John Wiley \& Sons. Inc; New York: 1989.

\section{Biographies}

H. Thomas Banks received his PhD in Applied Mathematics from Purdue University in 1967 and served on faculty in the Division of Applied Mathematics at Brown University from 1967 through 1989. From 1989 to 1992 he was on the faculty at the University of Southern California where he founded and served as the first Director of the Center for Applied Mathematical Sciences. In 1992 he joined the faculty at N.C. State University in Raleigh where he currently Distinguished University Professor and Drexel Professor of Mathematics and Director of the Center for Research in Scientific Computation. His research achievements include over 350 published papers in applied math, biology and engineering journals, elected Fellow of IEEE, elected Fellow of Institute of Physics, recipient of the 1996 IEEE Control Systems Technology 
Award, the 2002 SIAM W.T. \& Idalia Reid Prize in Applied Mathematics, as well as other research related honors and awards. His major research interests include inverse problems and control theory for differential, partial differential and delay equation systems, as well as applications in biology, mechanics, and engineering systems.

Sava Dediu received his diploma in Mathematics from Alexandru Ioan Cuza University, Iasi, Romania, in 1997. He earned an MS and a PhD in Applied Mathematics from Rensselaer Polytechnic Institute, Troy, NY in 2002 and 2005, respectively. Between August 2005 and August 2006 he was a Postdoctoral Fellow at the Statistical and Applied Mathematics Institute, RTP, NC. Currently, he is a Postdoctoral Research Associate at the Center for Research in Scientific Computation, North Carolina State University, Raleigh, NC. His main research interests are inverse problems for partial differential equations and sensitivity analysis for dynamical systems.

Hoan Kim Nguyen received her BS, MS and PhD degrees in Mathematics in 1999, 2000 and 2004, respectively, all from Virginia Polytechnic Institute and State University, Blacksburg, VA. From June, 2000, through December, 2000, she was employed at the Naval Research Laboratory where she worked on developing algorithms to track radar signals. From August, 2004, through August, 2005, she was a Postdoctoral Fellow at the Statistical and Applied Mathematics Institute, RTP, NC, and from September, 2005, through August, 2006, she was a Postdoctoral Research Associate at the Center for Research in Scientific Computation, North Carolina State University, Raleigh, NC. In September, 2006, she joined the Center for Naval Analyses as a Research Analyst in the Advanced Technology and System Analysis (ATSA) Division. Her research interests include computational mathematics, sensitivity analysis and delay differential equations. 


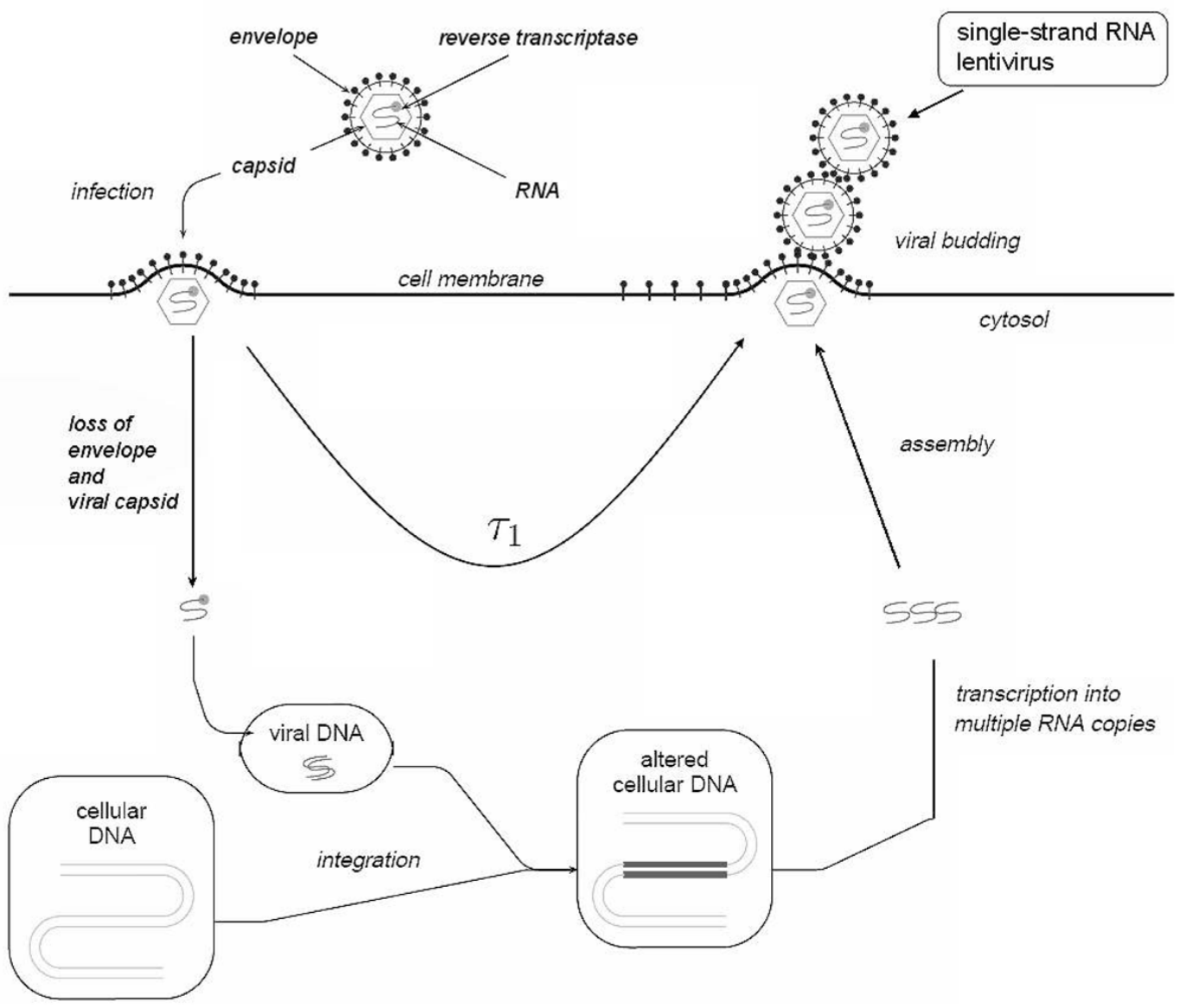

Figure 1.

HIV infection pathway in acutely infected cells. 